Geosci. Instrum. Method. Data Syst. Discuss., https://doi.org/10.5194/gi-2018-48

Manuscript under review for journal Geosci. Instrum. Method. Data Syst.

Discussion started: 6 March 2019

(c) Author(s) 2019. CC BY 4.0 License.

(c) (i)

Geoscientific

Instrumentation

Methods and

Data Systems

Discussions

\title{
Development of a New Centralized Data Acquisition System for Seismic Exploration
}

Feng Guo ${ }^{1}$, Qisheng Zhang ${ }^{1}$, Qimao Zhang ${ }^{2}$, Wenhao Li $^{1}$, Yueyun Luo ${ }^{1}$, Yuefeng Niu ${ }^{1}$, Shuaiqing Qiao ${ }^{1}$

$5 \quad$ School of Geophysics and Information Technology, China University of Geosciences Beijing, Beijing, 100083, China

${ }^{2}$ Institute of Electronics, Chinese Academy of Science, Beijing, 100190, China

Correspondence to: Qisheng Zhang(zqs@ @ cugb.edu.cn)

Abstract. Seismic exploration equipment has developed rapidly over the past few decades. One such piece of equipment is a centralized seismograph, which plays an important role in engineering, so improving its performance is of great scientific

10 significance. However, there is still a gap between seismic exploration equipment that is independently developed by China and that developed worldwide; this gap needs to be bridged via the advancements made in technology. In this research, the core part of general seismic data acquisition devices is packaged to develop a centralized seismic data acquisition system (Named as CUGB-CS48DAS) that has independent operating ability and high scalability, which can be used for engineering seismic and electrical prospecting. The low-power-consumption computer of the system comprises a 24-bit $\Sigma$ - $\triangle$ modulation

$15 \mathrm{~A} / \mathrm{D}$ converter and 48 sampling channels with an optional sampling rate of $50 \mathrm{~Hz}$ to $64 \mathrm{KHz}$, crosstalk rejection ratio $\geq 80$ $\mathrm{dB}$, dynamic range $\geq 120 \mathrm{~dB}$, frequency response range of $\mathrm{DC}$ to $16 \mathrm{KHz}$, synchronization accuracy better than $200 \mathrm{~ns}$, and data transmission speed $\geq 90$ Mbps. With regard to the host computer, the architecture of the control software is smart, and it can integrate the multiple functions of data acquisition, preprocessing, and self-testing; clear interfaces reduce the complexity of development and migration.

Keywords: Centralized Seismograph, Seismic Exploration, Electrical Prospecting, Function Extension, Data Acquisition, NB-IoT

\section{Introduction}

The development of seismic instruments has relied heavily on the continuous development of seismic data acquisition methods(S. Q. Qiao et al., 2018). Over the last 80 years, seismic exploration instruments have undergone five major developments: the electron tube (optical spot recorders), transistors (analog tape recorders), conventional digital seismographs (digital tape recorders), 16-bit telemetry seismographs, and 24-bit telemetry seismographs(K. Z. Song et al., 2012). At the beginning of the 21st century, ION and Sercel launched the IV system and the 400 series of fully digital seismographs, respectively, which were recognized as sixth-generation seismographs in the industry(Q. S. Zhang et al., 2013). The development of seismic instruments cannot be isolated from the continuous development of seismic-data acquisition methods, combined with the latest technology available at the time. Currently, the development of seismographs 
Geosci. Instrum. Method. Data Syst. Discuss., https://doi.org/10.5194/gi-2018-48

Manuscript under review for journal Geosci. Instrum. Method. Data Syst.

Discussion started: 6 March 2019

(c) Author(s) 2019. CC BY 4.0 License.

(c) (i)

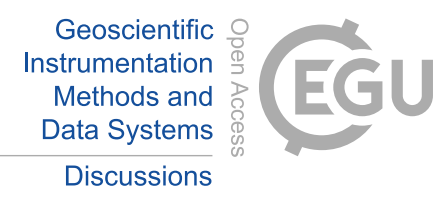

is highly dependent on the progress of electronic technology(X. Zhao et al., 2015), computer science, seismic exploration methods, intelligent control, network technology(G. Liang and W. Li, 2016), signal processing, and other disciplines(Q. S. Zhang et al., 2012), this development mutually reinforces the progress of the aforementioned disciplines.

5 The change from analog circuits to digital circuits has significantly increased the sampling accuracy, dynamic range, passband width, and number of data channels(S. Nakagawa, 2011). In terms of hardware circuitry, the presence of an analog-digital conversion acquisition chip and the high-performance conditioning filter circuit allows for a theoretical sampling precision of up to 24 bits and instantaneous dynamic range of more than $120 \mathrm{~dB}$. In terms of software, the automation of instrument operation allows for simple and convenient data processing.

10

The integrated framework of the centralized seismograph is usually fixed after entering the digital stage. Consider for example engineering seismographs such as SmartSeisST and ES-3000 from Laurel Technologies, whose major structure comprises a sensor (detector) receiving end, a signal conditioning circuit, an analog-digital conversion circuit, a control logic, and a human-computer interface (HCI) along the direction of data flow transmission(S. Mazza et al., 2012). Therefore,

15 the major structure of the centralized seismograph is relatively stable. The difference between various models lies in the auxiliary equipment (printer, built-in GPS, etc.), the channel number of the acquisition circuit, sample performance parameters, or instrument interface (Z. Q. Wu et al., 2011). Hence, we can separately package the conditioning circuit, the $\mathrm{AD}$ conversion circuit, the logic controller, and the software into a fixed core acquisition system. In this system, hardware and software can be customized and extended to acquire data via different seismic exploration methods, thus saving plenty of resources in the development of the instrument.

With this idea, a scalable data acquisition system (Named as CUGB-CS48DAS) is designed on the basis of the common PC104. The CUGB-CS48DAS data acquisition system can be used for seismic exploration as well as electrical prospecting. It has good application effects in engineering geology, mineral geology and energy geology, and is suitable for exploration tasks in coalfields, petroleum, minerals, earthquakes and urban construction, etc. In particular, with the introduction of the technology named Narrow Band Internet of Things (NB-IoT), it will be simpler to realize multi-system networking, which provides great convenience for unified monitoring and management of the acquisition instruments during the exploration.

This paper focuses on describing the hardware and software architecture of the CUGB-CS48DAS system, as well as its implementation mechanism. The feasibility of this system is then demonstrated by using a specific case. 
Geosci. Instrum. Method. Data Syst. Discuss., https://doi.org/10.5194/gi-2018-48

Manuscript under review for journal Geosci. Instrum. Method. Data Syst.

Discussion started: 6 March 2019

(c) Author(s) 2019. CC BY 4.0 License.

(c) (i)

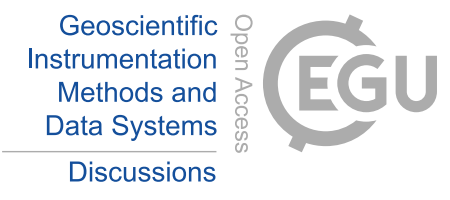

\section{Structure of the Acquisition System}

The development of the acquisition system generally involves hardware circuit design and control software development (D. Marjanovic et al., 2015). The CUGB-CS48DAS system structure is shown in Figure 1. Basic performance parameters and functions of the acquisition system depend on the hardware circuit, which is essential for ensure the quality of the acquired data. The operating state of the overall system is coordinated by the software, which is subject to certain fault tolerance and more flexible than the hardware circuit. It is required to encapsulate operations near hardware bottom and provide flexible and safe interfaces, thus both ensuring the system stability and improving its flexibility and portability.

\subsection{Hardware Architecture}

The acquisition system mainly comprises a host control module, a power module, an acquisition control module, and an 10 acquisition unit array, the CUGB-CS48DAS system structure is shown in Figure 1.

The host control module can implement system status control, data concentration, and human-computer interaction. The module mainly adopts a PC104 (model)-based embedded system with a Windows XP Embedded operating system and software written and compiled in MSVC. The host control module itself has two USB 2.0 interfaces, four RS232 serial ports,

15 one $100 \mathrm{M}$ network port, and one screen LVDS port. Two of the four serial ports are used by the host computer to control the operating state of the other modules. The other two ports are reserved for functional extensions. The $100 \mathrm{M}$ network is used for transmitting acquired seismic data. The LVDS data cables are connected to an external LCD screen with adjustable backlight brightness. The USB interfaces are connected to a Bluetooth peripheral as the input device of the instrument.

20 The power module is used to supply power to the system, monitor all module operating states, condition acquired synchronizing signals, etc. The module consists of an MCU circuit, a level switch circuit, and a synchronizing trigger circuit. The MCU circuit mainly consists of a C8051F320 microcontroller and related peripheral circuits. It can supply power to different system modules by strobing for relays of the level switch circuit. It also receives operating parameters of circuits via its own $\mathrm{AD}$ converter to monitor the operating states of modules. The level switch circuit can convert the external $12 \mathrm{~V}$ power into operating voltages required for the sub-modules of the acquisition system. The design needs to provide level switch stability to ensure normal operation of the acquisition system and accuracy of data acquisition. The synchronizing trigger circuit can isolate the acquired synchronizing signals, shape the signals, and then transmit them to the acquisition control module and the acquisition unit array to ensure temporal synchronization of channel data.

30 The acquisition control module can achieve logic control of the acquisition unit array and transmission of acquired data. The modular circuit mainly comprises a field-programmable gate array (FPGA) chip (EP3C25F324I7N) and a $100 \mathrm{M}$ network chip (DM9000). The module function mainly relies on the FPGA. A soft CPU is set up on the FPGA by using system-on-a- 
Geosci. Instrum. Method. Data Syst. Discuss., https://doi.org/10.5194/gi-2018-48

Manuscript under review for journal Geosci. Instrum. Method. Data Syst.

Discussion started: 6 March 2019

(c) Author(s) 2019. CC BY 4.0 License.

(c) (i)

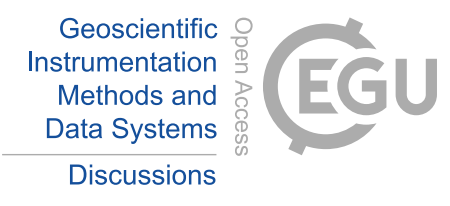

programmable-chip(SoPC) technology to communicate with other modules. The circuit module written in VHDL can provide data transmission between modules.

The acquisition unit array comprises multiple acquisition units, each of which consists of one host controller FPGA and one 5 group of AD conversion circuits (ADS1274). The controller can achieve control of conversion circuits and communication for the acquisition control system by setting up an SoPC on the FPGA. The AD conversion circuit mainly comprises three 4channel AD converters (ADS1274) and relevant conditioning circuits.

\subsection{System Software Description}

The host computer software is designed on the basis of the MFC provided by Microsoft. The software framework in this

10 study is shown in Figure 2. The system software architecture comprises an interactive interface, background program, and embedded system. The drawing module, methods manager, and document manager of the interactive interface can be adjusted or replaced depending on actual method requirements. The process controller of the background program is the core of the software that provides a functional interface for interface software development.

15 The process controller is mainly designed to enable the host computer control system to communicate with the power supply and the acquisition control system, and it can complete a full set of acquisition processes based on different methods and monitor the operating states of the instrument parts. The process controller comprises three basic units (a power control unit, a data acquisition control unit, and a data transmission unit) to provide services. Each unit consists of three layers, i.e., the implementation layer, protocol layer, and control layer. The implementation layer facilitates communication between the

20 software and underlying operating system. The protocol layer can parse data of the drive layer to find corresponding commands or data in the protocol table. The control layer, as a functional interface for the above two layers, can transmit messages corresponding to commands, so that each module can complete a series of tasks under the management of the process manager.

25 The exploration methods manager uniformly converts exploration methods selected on the HCI and relevant parameters to a data structure that can be identified between software modules and that can ensure parameter synchronization between modules.

The document manager can read and format raw data. Format templates for data storage are selected based on specific exploration methods. For example, seismic exploration methods are converted to SEG-2, SEG-Y, and other formats.

The drawing module is used for the graphical display of data. It is designed to manage acquisition parameters and actual data blocks, as well as handle graphic display operations for users. 
Geosci. Instrum. Method. Data Syst. Discuss., https://doi.org/10.5194/gi-2018-48

Manuscript under review for journal Geosci. Instrum. Method. Data Syst.

Discussion started: 6 March 2019

(c) Author(s) 2019. CC BY 4.0 License.

(c) (i)

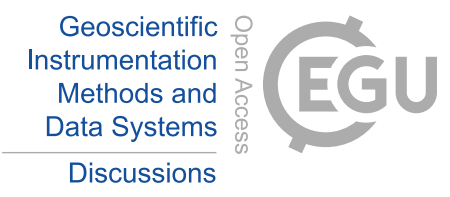

\section{Core Technology for Acquisition System}

It is critical for the overall acquisition system to achieve complete, stable, and portable acquisition workflow. In the acquisition process, the process controller of the host control module plays an important role. In addition to defining communication protocols and workflows between modules to determine the operating state of the overall system, the process

5 controller also acts as the hardware emulation layer for architecture development, provides a complete application interface for the software interface of the instrument, which is essential for the overall acquisition system to possess high project portability.

\subsection{Packaging Design of Process Controller}

The packaging design of a process controller is shown in Figure 3. The process controller can provide a data interface, functional interface, and message interface for the host control system software.

When the process controller works, multiple threads and tasks will start simultaneously and data and message communication will be required between the functional layers and between the sub-modules. In order to ensure normal software operation, communication between threads under the same sub-module should be regulated via a synchronizer and communication between functional layers should be regulated via a message stack. Part of the message stack is packaged as an interface to facilitate flexibility of the process controller.

The data interface partitions part of the memory area in service as the hard disk (HD) area by using RAMDisk. The user interface is in essence used for the mapping of cache files in the memory area. It can ensure high access speed of cache files and security in file sharing.

The functional interface mainly provides performance functions and public variables for operating the process controller, such as the sub-module state inquiry and configuration.

25 The message interface comprises a process message table and message allocation threads. The process message table is subject to the fixed priority setting, to collect and distribute messages of the process controller at work. Generally, error messages are at top priority, followed by network data-related messages, with a view to ensure the instrument stability and high transmission rate of acquired data. The send message from the serial port is at low priority, followed by a return message. It involves operating the relay of the lower computer and other hardware configured with a low speed ( $\geq 100 \mathrm{~ms})$,

30 so the requirement for response time is low. Each sub-module has its own message stack. Communication between submodules and that between sub-module and the process controller are assigned via a message interface at the upper level. 
Geosci. Instrum. Method. Data Syst. Discuss., https://doi.org/10.5194/gi-2018-48

Manuscript under review for journal Geosci. Instrum. Method. Data Syst.

Discussion started: 6 March 2019

(c) Author(s) 2019. CC BY 4.0 License.

(c) (i)

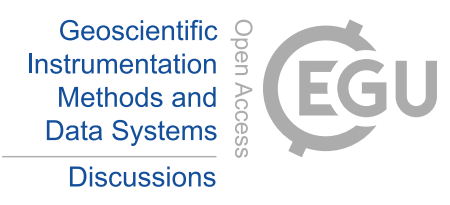

\subsection{System Acquisition Process Design}

Single data acquisition is the most basic unit for system acquisition. Other acquisitions of the system are achieved by adjusting or calling the single data acquisition.

5 For each acquisition, the power of the acquisition control module and the acquisition unit array has to be turned on, wait for the termination of initialization, and then configure acquisition parameters for the acquisition control module. For formal acquisition, a command shall be sent to the acquisition control module to make it ready and return a message to the host computer; the host computer then enters the data receiving mode. The acquisition control module transmits data to the host computer via the network. After the host control system receives network data packages, the data transfer unit will receive

10 and process the data package. After the acquisition is completed, the data transfer unit will enter the preprocessing mode to collate and cache the data. Next, the acquisition mode is determined. The data will be processed by self-test procedures to obtain a self-test report when the instrument self-test is conducted. For normal acquisition, the data will be sent to the document manager and the drawing unit for processing. When the continuous acquisition is completed, the process controller will reset and turn off the acquisition control module and the acquisition unit array.

15

In the acquisition system, the actual data transfer rate and the preset sampling rate share a linear relationship. The effective data transfer rate should reach $16 \mathrm{Mbps}$ for normal operation. Test results have shown that the data transfer rate between the acquisition control module and the data acquisition unit array can reach $100 \mathrm{Mbps}$ and that between the acquisition control module and the host control module can reach $90 \mathrm{Mbps}$, while the data receiving rate of the host control module is generally about 16 Mbps.

Data receiving in the host control module is a bottleneck for the entire system. Hence, the thread concurrency should be minimized in the single acquisition design in order to improve the data transfer rate and stability of the system. In particular, data receiving is separated from data feedback on the HMI, while the timing query of the message stack is used in the drawing part to confirm data acquisition progress and display it on the interface. After the abovementioned processing, the host control module can attain a data receiving rate of up to $90 \mathrm{Mbps}$, which can meet the demand of normal operation (16 Mbps).

\subsection{System Data Transmission Technology}

For the convenience of indoor data processing and interpretation of seismic data, field data collection accuracy has to be high,

30 and the bit error rate has to be low. To ensure the accuracy of data, the collected seismic data need to be encoded and then sent from the Acquisition Unit to the Acquisition Control Module. The transmission coding method of CUGB- CS48DAS 
Geosci. Instrum. Method. Data Syst. Discuss., https://doi.org/10.5194/gi-2018-48

Manuscript under review for journal Geosci. Instrum. Method. Data Syst.

Discussion started: 6 March 2019

(c) Author(s) 2019. CC BY 4.0 License.

(c) (i)

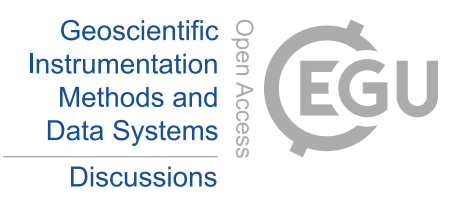

uses Manchester Encoding, which has the advantage of being able to extract the synchronization clock from the signal more easily.

The encoding process is completed in the Acquisition Unit, and the encoding module is written in VHDL. The parallel data

5 in bytes are converted into one-bit serial data which are encoded in the form of Manchester Encoding. During the encoding process, binary " 0 " is converted to " $01 "$ ", and binary " 1 " is converted to " $10 "$ ". Since the receiving end needs to align the valid edge with a certain period of time to achieve constant synchronization, a preamble must be sent before sending one frame of data. Then the encoded data are transmitted via LVDS which takes advantage of low noise and low power consumption

10 The Acquisition Control Module receives the data from Acquisition Unit through the LVDS interface. After receiving the preamble, the receiving end will complete the clock synchronization in several data cycles. The decoding module, which is also written in VHDL, uses a clock 8 times that of the data speed to detect the data jumps during the effective jumping time. Once the data have an early or a delayed transition, the decoding module automatically aligns with the new edge. In this way, the data can be allowed to shake to a large extent without causing bit errors. Besides, the decoding module also cope with

15 synchronized header determination and byte synchronization, etc. Eventually, the data are saved in the SDRAM and they can be acquired by the Host Control Module through 100 M network.

\section{Function Extension Examples}

As known from the system description, the overall system can independently perform basic functions such as instrument self-testing and data storage by using the process controller and the core hardware. Therefore, the process controller and core hardware can be packaged as a whole to provide technical support for other acquisition systems with different functions.

The porting of the acquisition system is accomplished in three steps. (1) The acquisition systems should be able meet project performance requirements. (2) In terms of hardware, the circuit board size and the layout of device connectors and modules should be adjusted. (3) In terms of software, the process controller interfaces should be compiled with the interactive interface, methods manager, document manager, and drawing module.

It is easy to implement this method technically. In terms of hardware, the logic control part is included in the FPGA chip with a generally fixed interface, and it can be easily upgraded. In terms of software, the process controller is as assumed to be an independent linkbase. The methods manager, the document manager, and the drawing module can use it for joint

30 compilation. Even the scripting language is used to provide an interactive interface function, thus greatly enhancing system reusability. 
Geosci. Instrum. Method. Data Syst. Discuss., https://doi.org/10.5194/gi-2018-48

Manuscript under review for journal Geosci. Instrum. Method. Data Syst.

Discussion started: 6 March 2019

(c) Author(s) 2019. CC BY 4.0 License.

(c) (i)

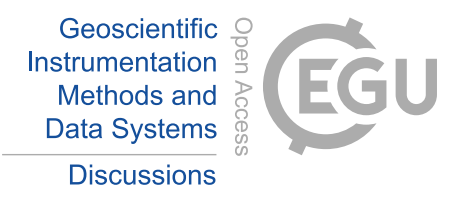

The acquisition system has been verified in actual engineering applications. In addition, some cases are employed to demonstrate great scalability of the system.

\subsection{Application of Centralized Seismic Exploration Instrument}

CUGB-CS48DAS is an engineering seismic exploration instrument obtained by customizing certain functions of the core

5 acquisition system. The monitoring mechanism of the power module and data acquisition mechanism of the acquisition control part are optimized. Nine common engineering seismic exploration methods such as coverage measurement, reflection, refraction, and surface wave are included in the method selector of the host control system. Some interfaces of the drawing module and the document manager are modified.

10 The instrument was used for a two-week seismic exploration in an area of Golmud City, Qinghai Province in 2013; the total measuring line length was $4.4 \mathrm{~km}$, the seismic migration profile is shown in Figure 4. The exploration adopted the CDJZ/P60 geophone of the Chongqing Instrument Factory and explosives as a seismic source. The sampling rate was $4 \mathrm{kHz}$, with 2048 sampling points and 24 channels. Seismic surveying utilizes the coverage measurement method.

\section{$15 \quad 4.2$ Realization of Distributed Seismic Exploration Instrument}

CUGB-CS48DAS was designed to provide additional distributed acquisition station functions on the basis of 48 channels to the original system and achieve data transfer and command control via network cables. Distributed control was added to the acquisition control system on the hardware layer. In the host control system, the operating interface, the drawing module, and the document manager were modified and 48 sampling channels were extended to 96 , the system diagram is shown in

20 Figure 5, connect CUGB-CS48DAS and Distributed Sampling Unit with PoE (Power Over Ethernet).

\subsection{Extension of Electrical Prospecting}

In August 2014, an extension of the electrical prospecting function was achieved on the basis of the CUGB-CS48DAS seismograph. The extension of geophysical electrical prospecting is shown in Figure 6. An electrical prospecting controller and an electrical transmitter were added to the original system, and sensors were changed to electrodes. The host computer of the original system was directly connected to the electrical prospecting controller via serial ports. The overall acquisition process can be controlled using the electrical prospecting controller. The electrical prospecting controller can control the electrode channel configuration and operating state of the electrical transmitter based on host computer commands. The main part of the electrical transmitter is the medium-power generators used for geophysical electrical prospecting of the measured area, so that the measured objects show electrical characteristics (such as apparent resistivity and apparent polarizability). By

30 controlling the electrical transmitter and changing the frequency and waveform of the emission current, the measurements of the induced polarization method in both frequency domain and time domain can be performed. 
Geosci. Instrum. Method. Data Syst. Discuss., https://doi.org/10.5194/gi-2018-48

Manuscript under review for journal Geosci. Instrum. Method. Data Syst.

Discussion started: 6 March 2019

(c) Author(s) 2019. CC BY 4.0 License.

(c) (i)

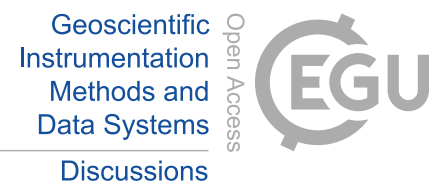

\subsection{Connection to the Internet of Things}

Recently, the NB-IoT chip named BC95 was introduced to make it possible for connecting the Internet of things. Via the UART interface, data exchange between the host control module and BC95 can be accomplished. Commercial network

5 become available thanks to the internal GSM/GPRS module of BC95.That means the CUGB-CS48DAS acquisition system has the ability to connect to the NB-IoT network as convenient as normal mobile phones connecting to the Internet. As is illustrated in Figure 7, data from host control module are sent to the BC95 through the UART interface, then uploaded to the cloud server using the NB-IoT network. The data could be information of sensors or states of acquisition process, etc. Conversely, commands may also be sent to the CUGB-CS48DAS from cloud server to control the acquisition process.

10 Depending on the NB-IoT network erected by operators, ideally, a large number of acquisition systems distributed throughout a wide open area will be monitored at the same time. As a result, the CUGB-CS48DAS can now be used to detect the urban underground space for a long-term purpose while being controlled at meantime because of the low power consumption of NB-IoT technology.

\section{Data Acquisition System Performance Indicators}

15 After conducting tests and analysis in the laboratory and in the field, the acquisition system developed has the following performance indicators:

(1) An embedded Windows XP data acquisition interface that supports methods of surveying coverage measurement, reflection, and refraction. The system will be able to expand the collecting function of the geophysical electrical prospecting.

(2) Low-pass filtering: $0.8 \mathrm{FN}$ (digital filter);

20 (3) Sampling rate: $50 \mathrm{~Hz}$ to $64 \mathrm{KHz}$;

(4) Word length: 24 bits;

(5) Power supply: DC 12 V;

(6) Dynamic range: $120 \mathrm{~dB}$ at $0.4 \mathrm{KHz} \mathrm{BW}$ (1 KHz sampling rate);

(7) Total harmonic distortion: $-105 \mathrm{~dB}$;

25 (8) CMRR: > $102 \mathrm{~dB}$;

(9) Stop-band attenuation >120 dB (above the Nyquist frequency);

(10) Noise (DC to $200 \mathrm{~Hz}$ ): $450 \mathrm{nV}$ RMS at $0 \mathrm{~dB}$;

(11) Data transmission speed: $90 \mathrm{Mbps}$;

(12) Synchronization accuracy: better than $200 \mathrm{~ns}$;

30 (13) Operating temperature: $-20^{\circ} \mathrm{C}$ to $+70^{\circ} \mathrm{C}$. 
Geosci. Instrum. Method. Data Syst. Discuss., https://doi.org/10.5194/gi-2018-48

Manuscript under review for journal Geosci. Instrum. Method. Data Syst.

Discussion started: 6 March 2019

(c) Author(s) 2019. CC BY 4.0 License.

(c) (i)

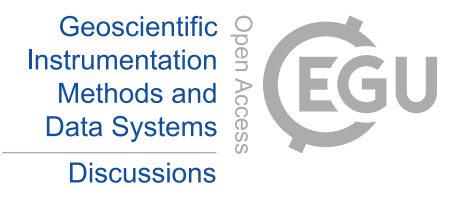

\section{Conclusions}

In this study, a new type of centralized data acquisition system was designed, based on the SoPC technique. Through a research and development program, the following technical aspects were explored:

5 (1) In accordance with the framework and actual results, CUGB-CS48DAS is a complete and independent acquisition system. Practical applications and extension cases show the system has better scalability and portability, with scope for use in many applications. Further standardization of the application interfaces of the system is expected, along with a reduction in cost. We will attempt to apply this system to data acquisition tasks besides geophysical exploration.

10 (2) The seismic exploration data acquisition and processing technology using the SoPC technique as the master control core: this technology introduces the most recent SoPC technique into engineering seismic exploration, which reduces power consumption while improving seismograph resolution and synchronization accuracy. The system can also be flexibly embedded into the seismic exploration data processing algorithm in the form of hardware.

15 (3) 48-channel, 24-bit high-precision parallel $\mathrm{AD}$ conversion technique: the multi-channel seismic data acquisition performed in existing engineering seismic exploration uses only $1 \mathrm{AD}$ converter, resulting in poor synchronization when carrying out time-shared data acquisition; whereas in this design, every channel uses a separate high-precision AD converter to provide synchronization precision.

20 (4) CUGB-CS48DAS can solve the multi-solution problem in geophysical prospecting and achieve joint geophysical seismic and electrical prospecting. The single method of present geophysical prospecting has a multi-solution problem and thus cannot be refined; nevertheless, this new centralized data collection system provides technical means to solve this problem while offering useful explorations for joint geophysical seismic and electrical prospecting. However, the increase of synchronization precision and acquisition accuracy provides technical support for more refined seismic prospecting, yet

25 because the increase of synchronization precision and acquisition accuracy has met the requirements of high-precision geophysical electrical prospecting, novel supporting equipment has been employed for joint geophysical seismic and electrical prospecting.

(5) The introduction of embedded Windows XP technology has improved the human-machine interactive interface of an 30 engineering seismograph. 
Geosci. Instrum. Method. Data Syst. Discuss., https://doi.org/10.5194/gi-2018-48

Manuscript under review for journal Geosci. Instrum. Method. Data Syst.

Discussion started: 6 March 2019

(c) Author(s) 2019. CC BY 4.0 License.

(c) (i)

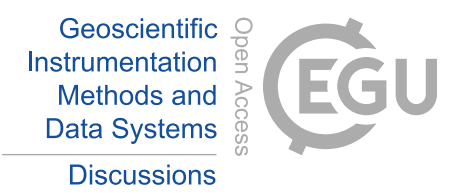

\section{Funding Acknowledgement}

This work is supported by the Natural Science Foundation of China (No. 41574131 and No. 41204135), the National "863" Program of China (No. 2012AA061102 and No. 2012AA09A20102), the National Major Scientific Research Equipment Research Projects of China (No. ZDYZ2012-1-05-01), and the Fundamental Research Funds for the Central Universities of 5 China (No. 2652015213).

\section{References}

D. Marjanovic, M. Grozdanovic, G. Janackovic: Data acquisition and remote control systems in coal mines: a serbian experience, J. Mea. Control, vol.48, pp.28-36, Feb. 2015.

G. Liang, W. Li: Some thoughts and practice on performance improvement in distributed control system based on fieldbus and ethernet, J. Mea. Control, vol.49, pp.109-118, April. 2016.

K. Z. Song, G. P. Cao, J. F. Yang and P. Cao: A high-precision synchronous sampling approach for large-scale distributed Wire sensor networks in seismic data acquisition systems, J. Instrum. Sci. Technol., vol. 40, pp. 567-579, 2012.

Q. S. Zhang, M. Deng, J. Guo, W. B. Luo, Q. Wang and Y. Q. Feng: Development of a new seismic-data acquisition station based on system-on-a-programmable-chip technology, J. Ann. Geop., vol. 56, pp. 184-190, 2013.

15 Q. S. Zhang, M. Deng, J. L. Cui and Q. Wang: Research and development of one novel distributed digital seismic acquisition station, J. Adv. Inf. Sci. Serv. Sci., vol. 4, pp. 184-190, 2012.

S. Mazza, A. Basili, A. Bono, V. Lauciani, A.G. Mandiello, C. Marcocci, F. Mele, S. Pintore, M. Quintiliani, L. Scognamiglio and G. Selvaggi.: AIDA-Seismic data acquisition, processing, storage and distribution at the National Earthquake Center, J. Ann. Geop. 55(4), pp. 541-548, 2012.

20 S. Nakagawa: Split Hopkinson resonant bar test for sonic-frequency acoustic velocity and attenuation measurements of small, isotropic geological samples, J. Rev. Sci. Instrum. 82(4), 044901, 2011.

S. Q. Qiao, H. M. Duan, Q.S. Zhang: Development of high-precision distributed wireless microseismic acquisition stations, J. Geosci. Instrum. Method. Data Syst., published online, doi: 10.5194/gi-2018-17, 2018.

X. Zhao, Q. S. Zhang and M. Deng: Super class-AB bulk-driven OTAs with improved slew rate, J. Electron. Lett., vol. 51, pp. 1488-1489, 2015.

Z. Q. Wu, S.G. Wu, S.Y. Tong, H.S. Liu and Y.B. Zhang: A study on seismic acquisition basic on marine carbonate hydrocarbon exploration in the southern Yellow Sea, J. Chin. J. Geop. 54(4), pp. 1061-1070, 2011. 

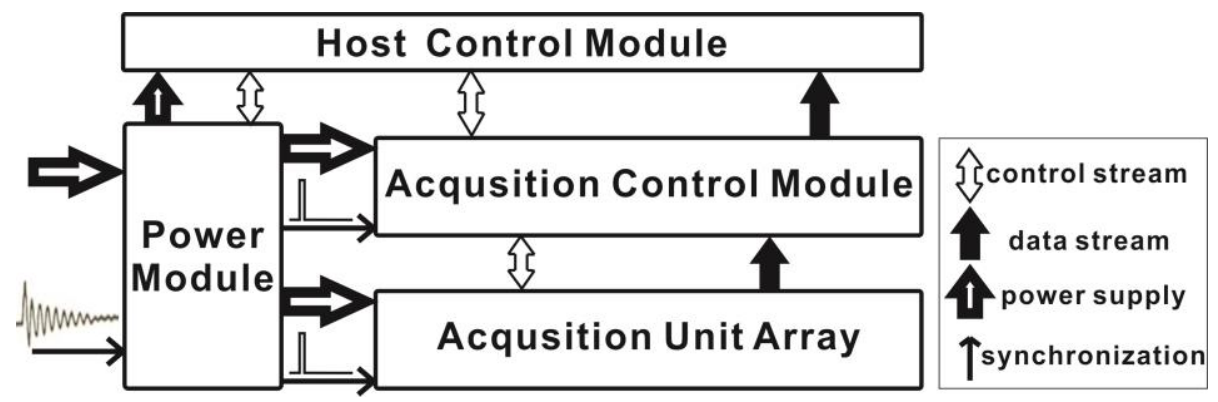

Figure 1: Structure diagram of CUGB-CS48DAS seismograph.

\begin{tabular}{|c|c|c|}
\hline \multicolumn{3}{|c|}{ Interactive Interface } \\
\hline \multicolumn{3}{|c|}{ Drawing Module } \\
\hline \multicolumn{3}{|c|}{35} \\
\hline \multicolumn{2}{|l|}{$\begin{array}{l}\text { Methods } \\
\text { Manager }\end{array}$} & $\begin{array}{l}\text { Document } \\
\text { Manager }\end{array}$ \\
\hline \multicolumn{3}{|l|}{ इ5 } \\
\hline \multicolumn{3}{|c|}{ Backstage Supporter } \\
\hline \multicolumn{3}{|c|}{ Process Controllers } \\
\hline $\begin{array}{l}\text { Power Control } \\
\text { Module }\end{array}$ & $\begin{array}{c}\text { Sampling Control } \\
\text { Module }\end{array}$ & $\begin{array}{l}\text { Data-Transfer } \\
\text { Module }\end{array}$ \\
\hline $\begin{array}{l}\text { Power Protocol } \\
\text { Stack }\end{array}$ & $\begin{array}{c}\text { Sampling Protocol } \\
\text { Stack }\end{array}$ & $\begin{array}{c}\text { Data Protocol } \\
\text { Stack }\end{array}$ \\
\hline \multicolumn{2}{|c|}{ Serial Ports Implementor } & NET Implementor \\
\hline \multicolumn{3}{|c|}{ System } \\
\hline
\end{tabular}

Figure 2: Software architecture of host computer control system 
Geosci. Instrum. Method. Data Syst. Discuss., https://doi.org/10.5194/gi-2018-48

Manuscript under review for journal Geosci. Instrum. Method. Data Syst.

Discussion started: 6 March 2019

(c) Author(s) 2019. CC BY 4.0 License.

Geoscientific

Instrumentation

Methods and

Data Systems

Discussions

(c) (1)

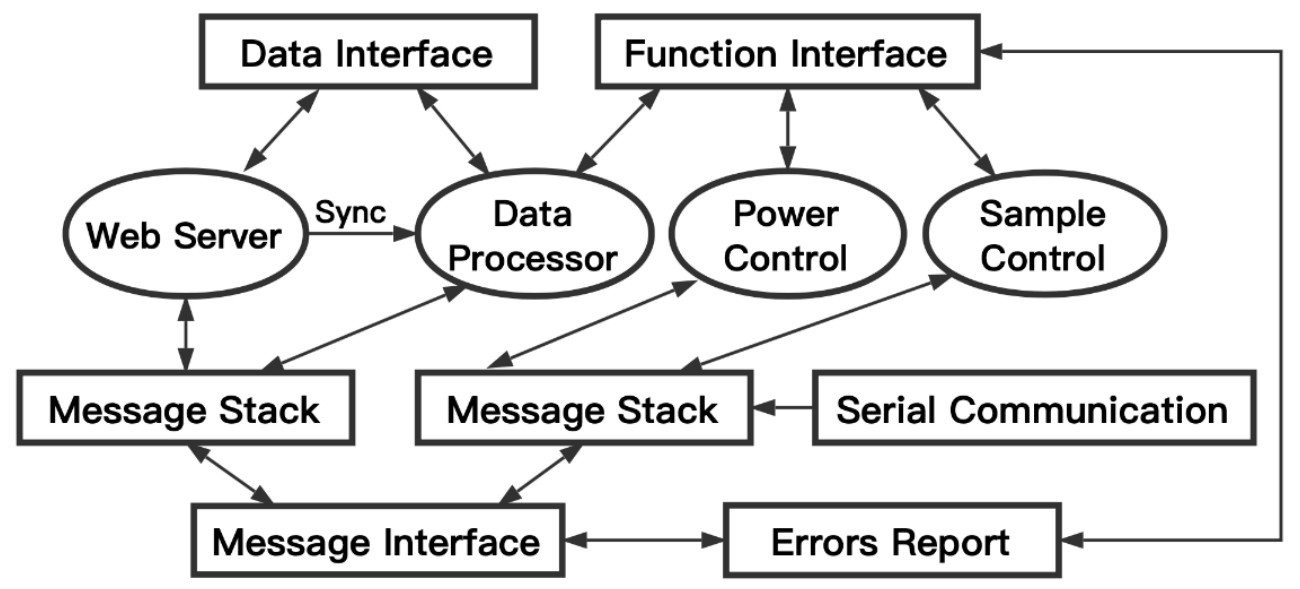

Figure 3: The packaging design of a process controller

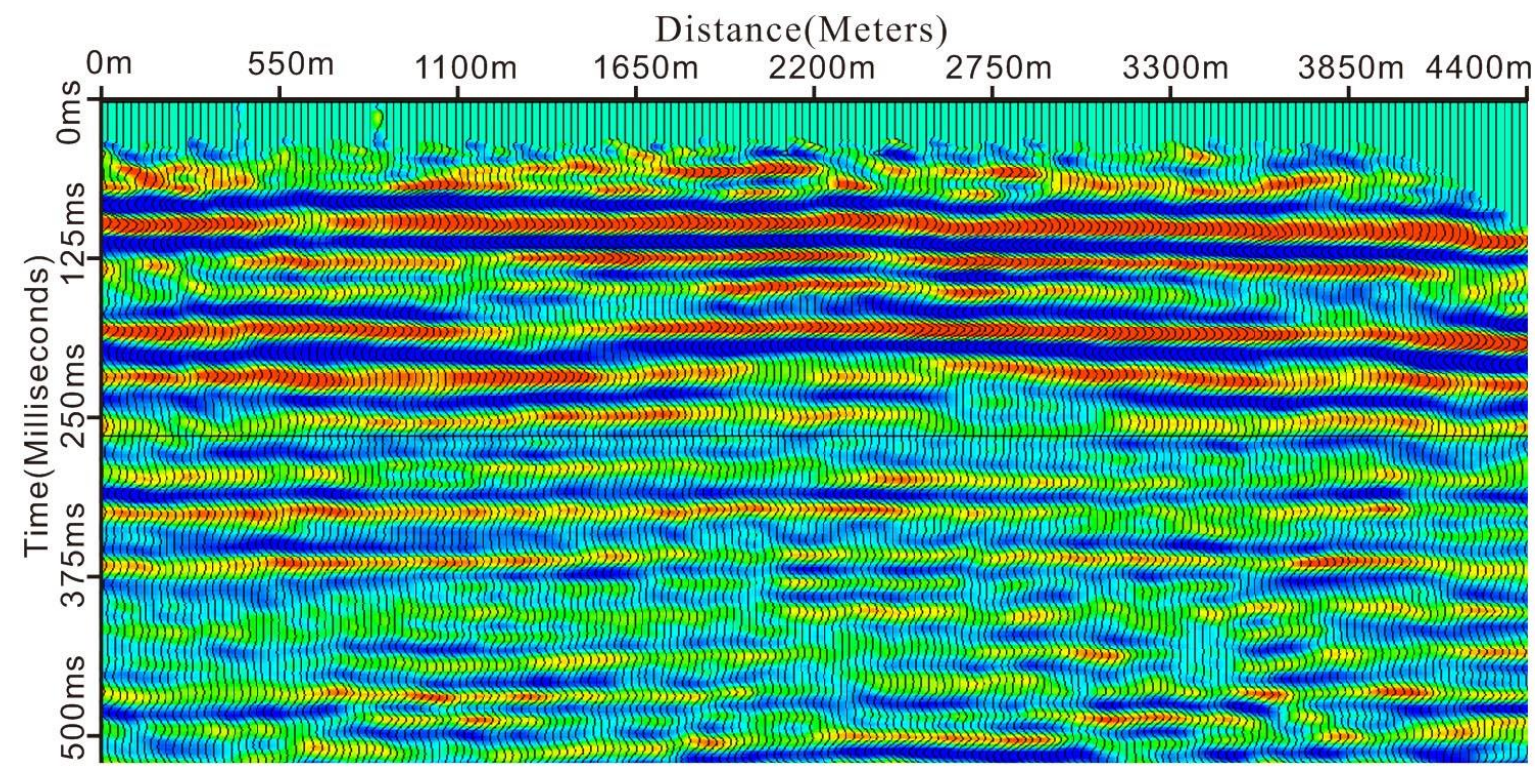

Figure 4: Seismic migration profile

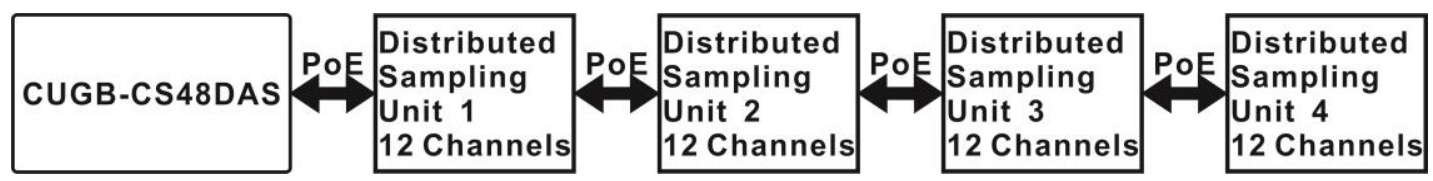

Figure 5: Distributed and centralized seismograph 
Geosci. Instrum. Method. Data Syst. Discuss., https://doi.org/10.5194/gi-2018-48

Manuscript under review for journal Geosci. Instrum. Method. Data Syst.

Discussion started: 6 March 2019

(c) Author(s) 2019. CC BY 4.0 License.

(c) (i)

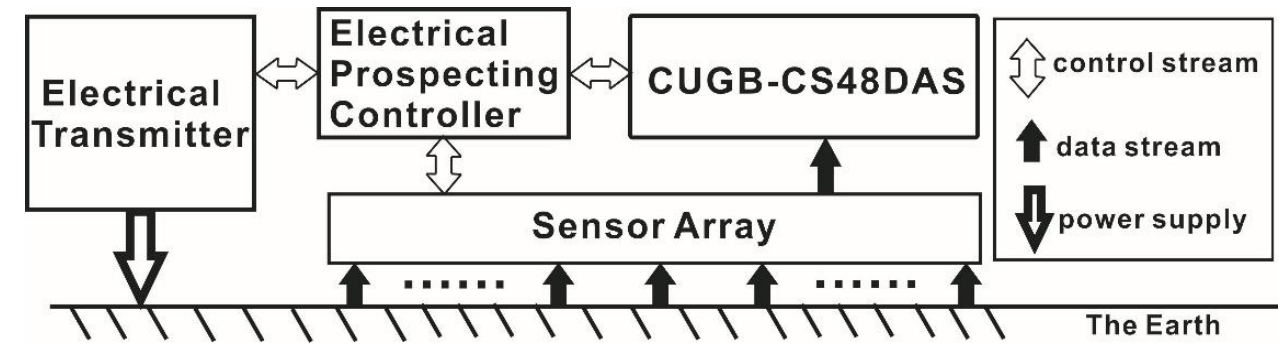

Figure 6: Extension of geophysical electrical prospecting

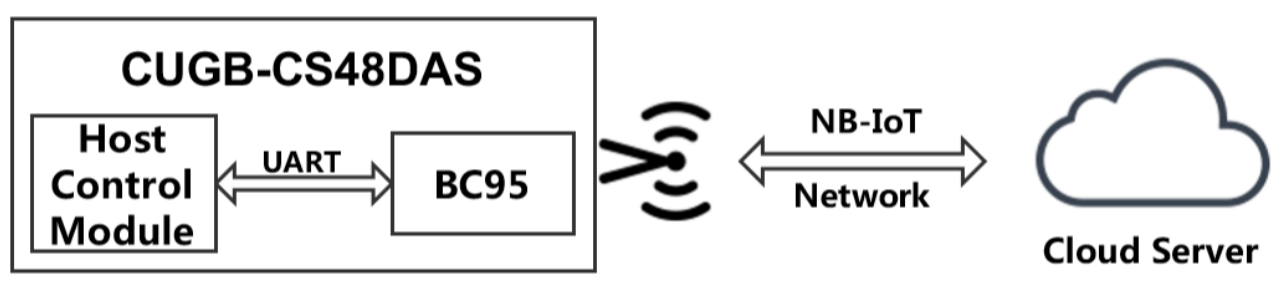

Figure 7: Connection to the Internet of Things 